\title{
Gastric Tubular Adenocarcinoma
}

National Cancer Institute

\section{Source}

National Cancer Institute. Gastric Tubular Adenocarcinoma. NCI Thesaurus. Code C5473.

A variant of gastric adenocarcinoma characterized by prominent dilated or slit-like tubules. 